\title{
The Lorentz-Dirac Equation and the Structures of Spacetime
}

\author{
Manoelito M. de Souza \\ Universidade Federal do Espírito Santo \\ Departamento de Física \\ 29065.900, Vitória, ES, Brasil \\ Received 11 November, 1997
}

\begin{abstract}
An interpretation of the causality implementation of the Lienard-Wiechert solution raises doubts against the validity of the Lorentz-Dirac equation and the limits of validity of the Minkowski structure of spacetime.
\end{abstract}

\section{Introduction}

Early in this century, the search for the correct equation of motion for a pointlike charged classical particle was a major problem in theoretical physics. The advent of quantum mechanics brought some hope that it could be properly understood in the framework of a quantum theory. The proposed third-order LorentzDirac equation could not be accepted because of its numerous problems (which have not been solved, but just forgotten). In our opinion, this was a bad point for theoretical physics: one has failed to see that the Minkowski space is not the appropriate underlying geometric structure for the description of close interacting fields. The solution of these problems is still of great relevance since it may signal steering corrections one has to make in field theory for avoiding old problems of QED and the stalling situations in some areas as quantum gravity and QCD.

In modern field theories, Poincaré invariance is imposed, and the Minkowski space-time is taken as the appropriate scenario for describing non-gravitational phenomena. For electromagnetic fields in vacuum, far from charges, this has received confirmation from a solid experimental basis, but not for fields in a close vicinity of their sources. Even from a theoretical viewpoint, the question is not so clear: the problems faced by quantum field theories for dealing with fields defined in close neighboring points are well known. These difficulties are generally taken as indications of some failure in the quantum basis of the theories (or at least as an indication of the existence of some limits of validity). In this paper, we wish to emphasize that the same problem occurs in classical physics disguised on this old controversy about the correct equation of motion for the classical electron. Having inherited the same spacetime structure of their classical predecessors, it is not surprising that the quantum theories also face a similar problem for defining fields in a close vicinity. Therefore, the roots of this problem must be searched at deeper grounds, in the very foundation of the assumed structures of the space-time continuum.

Assuming the validity of energy momentum conservation $[1,2,3]$, a classical spinless point charge in an isotropic and homogenous Poincaré invariant spacetime leads to the Lorentz-Dirac equation,

$$
m a=e F_{e x t} . V+\frac{2 e^{2}}{3}\left(\dot{a}-a^{2} V\right) .
$$

This equation is written in a context where the electron world-line, parameterized by its proper time $\tau$, is a known function, $z(\tau)$. Then, the electron velocity is $V=d z / d \tau ; \quad a=d V / d \tau$, and $\dot{a} \equiv d a / d \tau . e F_{\text {ext }}$ is the exterior force driving the electron, which, if taken as of electromagnetic origin, is written as $F_{e x t}^{\mu}=F_{e x t}^{\mu \nu} V_{\nu}$, where $m$ and $e$ are the electron mass and charge, respectively, and the speed of light is $c=1$. The presence of the Schott term, $\frac{2 e^{2}}{3} \dot{a}$, is the cause of some pathological 
features of (1), like microscopic non-causality, runaway solutions, preacceleration, and other bizarre effects [4]. The adoption of an integral equation with a convenient choice of limits can avoid either one of these two last problems, but not both. On the other hand, this term is necessary for energy-momentum conservation; without it would be required a null radiance $\left(a^{2}=0\right)$ for an accelerated charge. The argument, although correct, that such causality violations are not observable because they are outside the scope of classical physics [5] and are blurred [6] by quantum-mechanics effects is not enough compelling, because the same problems remain in a quantum formalism, just disguised in other apparently distinct problems.

It must be added that the inclusion of spin and some extension or structure for the electron would be just a complication without a changing in the essence of the problem. Taking a spinless particle is a valid simplifying hypothesis since the point at stake is not that one must consider every property of the physical electron, but why one gets physically non-acceptable results if one starts from apparently good premises and uses only mathematically sound procedures. It can only mean that something in the premises or in the procedures is not as good as one thinks. The problems that appear in both classical and quantum theories when one has to consider the limit situation of two objects (the electron and its electromagnetic field, for example) defined on a same point are the crux of the question. For the classical electron the picture is quite clear: the energy-momentum conservation produces sound physical results in any region around the charge except at the position of the charge. It will be argued in the following that this is a strong indication of the breaking down of the validity of some accepted premises about the structure of the space-time: electron and photon require different local space-time structure. The failure of recognizing this result yields the equation (1). It amounts to requiring that the propagation of a massive object (the electron) attends the same constraint of the photon (a massless object).

The geometrization of a physical principle is a very useful tool because it assures its automatic implementation and allows that we concentrate our attention on other aspects of the problem we are studying. The Minkowski spacetime represents a geometrization of the relativistic requirement that the velocity of light be a universal constant. Despite its undisputed success through the theory of special relativity, in the interface between a field and its source it produces a violation of causality. By revisiting the Lienard-Wiechert solution, we review its implicit and geometric causality implementation and show that it implies a more complex spacetime than the Minkowskian one with its lightcone structure. This suggested new model of spacetime requires a revision of our concepts of field theories of interacting massive and massless fields and shows the weak points in the demonstrations of the Lorentz-Dirac equation.

\section{The Lorentz-Dirac Equation}

The derivation of the Lorentz-Dirac equation, with the use of techniques of distribution theory, can be roughly schematized [1] in the following way. The electromagnetic field $F_{\mu \nu}=A_{\mu, \nu}-A_{\nu, \mu}$ with $\partial . A \equiv \partial_{\mu} A^{\mu}=0$, satisfies the Maxwell's equations, $\square F=4 \pi J$, where J, given by,

$$
J(x)=e \int d \tau V \delta^{4}[x-z(\tau)],
$$

is the current for a point particle with electric charge e and four-velocity V. The Lienard - Wiechert solution $[3,5,7]$,

$$
A=\frac{e V}{\rho}, \quad \rho>0
$$

in terms of retarded coordinates, by which any spacetime point $\mathrm{x}$ is constrained with a particle world-line point $z(\tau)$ by

$$
R^{2}=0
$$

and $R^{0}>0$, with $R \equiv x-z(\tau) ; \quad \rho \equiv-V . \eta . R$, where $\eta$ is the Minkowski metric tensor, (with signature +2$). \quad \rho$ is the spatial distance between the point $\mathrm{x}$ where the electromagnetic field is observed and the point $z(\tau)$, position of the charge, in the charge rest frame at its retarded time. The total particle and field energy-momentum tensor, $T=T_{m}+\Theta$, consists of

$$
T_{m}=m \int d \tau V V \delta^{4}[x-z(\tau)]
$$




$$
\Theta^{\mu \nu}=\frac{1}{4 \pi}\left(F^{\mu \alpha} F_{\alpha}^{\nu}-\frac{\eta^{\mu \nu}}{4} F^{\alpha \beta} F_{\beta \alpha}\right),
$$

where $F=F_{\text {ret }}+F_{\text {ext }}$ is the retarded field added to any external electromagnetic field acting on the charge. It induces $\Theta=\Theta_{\text {ret }}+\Theta_{m i x}+\Theta_{e x t}$. We are omitting any mention to some messy calculations [1] related to the highly non integrable parts of $\Theta_{\text {ret }}$, which requires some renormalizations of $\Theta$ on the charge worldline.
The required momentum conservation,

$$
T^{\mu \nu}, \nu=0
$$

is satisfied without any problem at any point but $\rho=$ $0, \quad(x=z)$, where $\mathrm{T}$ is not defined. In order to handle the singularity at $\rho=0$, T must be treated not as just a function defined only at $\rho>0$ but as a distribution defined everywhere. Then, (5) is replaced by

$$
\int d x^{4} T^{\mu \nu}{ }_{, \nu} \phi(x)=-\int d x^{4} T^{\mu \nu} \phi, \nu=-\lim _{\varepsilon \rightarrow 0} \int d x^{4} T^{\mu \nu} \phi,{ }_{\nu} \theta(\rho-\varepsilon)=0,
$$

where $\phi(x)$ is an arbitrary differentiable function with a compact support and $\theta(x)$ is the Heaviside function, $\theta(x>0)=1, \theta(x<0)=0$. Another integration by parts gives

$$
\lim _{\varepsilon \rightarrow 0} \int d x^{4} \rho, \nu T^{\mu \nu} \phi(x) \delta(\rho-\varepsilon)=0
$$

which, after integration, produces, in the limit, the Lorentz-Dirac equation (1). We want to pin point a crucial passage (common to most derivation of this kind) in this procedure for posterior careful analysis: the limit $\varepsilon \rightarrow 0$, which represents a change from a point $\mathrm{x}$ where there is only electromagnetic field and no electric charge, $\rho>0$, to a point $z(\tau)$, instantaneous location of the electron, $\rho=0$.

\section{Geometry of Causality}

There is a beautiful and physically meaningful underlying geometry describing the structure of causality in relativistic classical electrodynamics, of which we will give here just a brief description. The Lienard-Wiechert solution (3) is an explicit function of $\mathrm{x}$ and of the retarded proper time of its source, $\tau_{\text {ret }}$, which is a solution of the constraint (4). When taking derivatives of functions of retarded coordinates, like (3), the differentiation of the constraint (4) must be considered and it implies $[7,9]$ on $R . d R=0$, then $R>(d x-V d \tau)=0$ and $R . d x+\rho d \tau=0$, or

$$
d \tau+K . d x=0
$$

where $K \equiv R / \rho=-\partial \tau / \partial x$. The effects of this constraint on derivatives of functions of retarded time, like A, can be automatically accounted for if each derivative is replaced by a directional derivative,

$$
\partial_{\mu} \longrightarrow \nabla_{\mu} \equiv \partial_{\mu}-K_{\mu} \partial / \partial \tau
$$

so that

$$
\left.\partial_{\mu} A(x)\right|_{\tau_{\text {ret }}}=\nabla_{\mu} A(x) .
$$

On the RHS of (10) $\mathrm{x}$ and $\tau$ are treated as 5 independent parameters; the restriction (8) among $\tau$ and $\mathrm{x}$ (present on the LHS) is transferred to $\nabla$. The geometric meaning of $\nabla$ is then quite clear; it is the possible derivative allowed by the restriction (8), that is, displacements on the hyperplane $d \tau+K . d x=0$ only. The constraints (4) and (8) have clear geometrical and physical meanings: the electron and its electromagnetic field must belong to and remain in a same lightcone; they represent, respectively, a global and a local implementation of the relativistic causality.

In the standard formalism, which we are reviewing, there is a clear distinction between the treatment given to the electron and the one given to its electromagnetic field. It is now convenient to adopt a notation where these distinctions are reduced to the essentially necessary. So, we change the notation, replacing $\mathrm{R}$ by $\Delta x$ 
and extending its meaning to be a change in the location of a physical object (particles, fields, etc). Therefore, the constraint $R^{2}=0$, valid for the electromagnetic field, is replaced by

$$
\Delta x^{2}=0
$$

showing, in an explicit way, that this massless field propagates keeping constant its propertime, $\Delta \tau=0$. K as a null vector, $K^{2}=0$, represents a lightcone generator, the direction of propagation of the electromagnetic field. Equation (4) or (11) can be seen as a restriction on the set of solutions of (8), and both (11) and (8), are constraints to be imposed on the propagation of massless objects. They make sense for the electromagnetic field and as such they have accordingly been used in section 2 for $\rho>0$, but they cannot be extended to the propagation of a massive object, like an electron.

The appropriate constraint, equivalent to (11) for an electron, has to be

$$
-(\Delta \tau)^{2}=\Delta x^{2}
$$

where $\Delta \tau$ is the variation of the electron propertime during its propagation along a distance $\Delta x$ (which, for the electron, now means $\Delta z$ ); likewise, the constraint (8) must be replaced by

$$
d \tau+V . d x=0
$$

and, similarly, a directional derivative corresponding to (9) is defined, replacing $\mathrm{K}$ by $\mathrm{V}$ :

$$
\partial_{\mu} \longrightarrow \nabla_{\mu} \equiv \partial_{\mu}-V_{\mu} \partial / \partial \tau
$$

The differences between (9) and (14) just reflect the distinct constraints on the propagation of massive and of massless physical objects.

We are now in condition to define the unifying geometric background that underlies equations (9-14). Consider all the physical objects (electrons, electromagnetic fields, etc) immersed in a flat 5 -dimensional space, $R_{5} \equiv R_{4} \otimes R_{1}$, whose line elements are defined by

$$
\left(\Delta S_{5}\right)^{2}=\Delta x^{M} \eta_{\mathrm{MN}} \Delta x^{N}=\left(\Delta S_{4}\right)^{2}-\left(\Delta x^{5}\right)^{2}=\Delta x . \eta \cdot \Delta x-\left(\Delta x^{5}\right)^{2}
$$

where $M, N=1$ to 5 . Immersed in this larger space, every physical object is restricted to a 4-dimensional submanifold, its spacetime, by

$$
-\left(\Delta x^{5}\right)^{2}=\Delta x^{2}
$$

$\left(\Delta S_{5}\right)^{2}=-2(\Delta \tau)^{2}$ for a physical object, always. In other words, the change $\Delta x^{5}$ of a physical object is restricted to be the change of its very propertime, $\Delta x^{5}=\Delta \tau$. This is a causality condition, standing for both (11) and (12). So, the constraints on the propagation of physical objects become restrictions on their allowed domain in $R_{5}$, that is in the definition of their allowed spacetime. (16) may be written, in an obvious notation, as

$$
(\Delta t)^{2}=(\Delta \tau)^{2}+(\Delta \vec{x})^{2}
$$

which defines a 4-dimensional hypercone in the local tangent space of $R_{5}$. See Fig.1. It is a CAUSALITY-
CONE, a generalization of the Minkowski lightcone. A lightcone, the domain of a massless physical object, is an intersection of a causality-cone and a 4-dimensional hyperplane defined by: $x^{5}=$ const. The interior of a lightcone is the projection of a causality-cone on such a $\left(x^{5}=\right.$ constant $)$-hyperplane. Each observer perceives an strictly $(1+3)$-dimensional world and his $\Delta x^{5}$ coincides with the elapsed time measured on his own clock, as required by special relativity; it represents his aging, according to his own clock.

This is in contradistinction to a Kaluza-Klein type of theory for unification of fields, which uses a spacelike fifth dimension and then needs a compactification mechanism to justify the non observability of $x^{5}$. The use of a timelike fifth coordinate is, of course, not new in physics. See for example the references $[8,9,10]$ and the references therein. 
A subtle detail must be observed. It is not correct that we are interpreting $x^{5}$ as a proper time; it is $\Delta x^{5}$, the variation of $x^{5}$ of a physical object, that is interpreted as the variation of its proper time, its aging. The propagation of physical objects, in this geometric setting, is restricted by the differential of (16), $\Delta \tau d \tau+\Delta x . d x=0$, or

$$
d \tau+f . d x=0
$$

$f . d x=f_{\mu} d x^{\mu}$, where $f=\frac{\Delta x}{\Delta \tau}$, and $\mathrm{f}$ is a timelike four-vector if $d \tau \neq 0$, or (extending (18) to include) a light-like four-vector if $d \tau=0$. (18) defines a family of four-dimensional hyperplanes parameterized by $\mathrm{f}$, tangent to and enveloping the causality-cone (17). (17) and (18) define a causality-cone generator whose tangent, projected on a $\left(x^{5}=\right.$ constant $)$-hyperplane, is f. A lightlike $\mathrm{f}$ corresponds to $\mathrm{K}$ of (8) while a timelike f stands for $\mathrm{V}$ of (13).

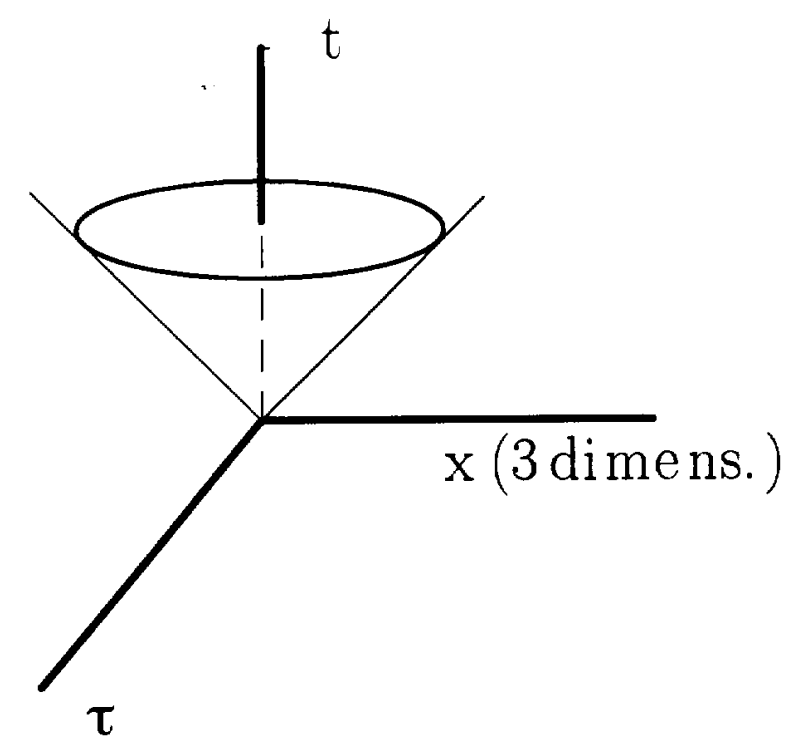

Figure 1. The Causality-cone.

The simultaneous imposition of (17) and (18) represents a causality implementation stricter than the Einstein causality (implemented through (11) only)). In the Einstein causality a physical object must remain inside (if $\Delta \tau \neq 0$ ) or on (if $\Delta \tau=0$ ) its light-cone. In this extended causality a free point physical object must remain on its causality-cone generator (its causality-line).

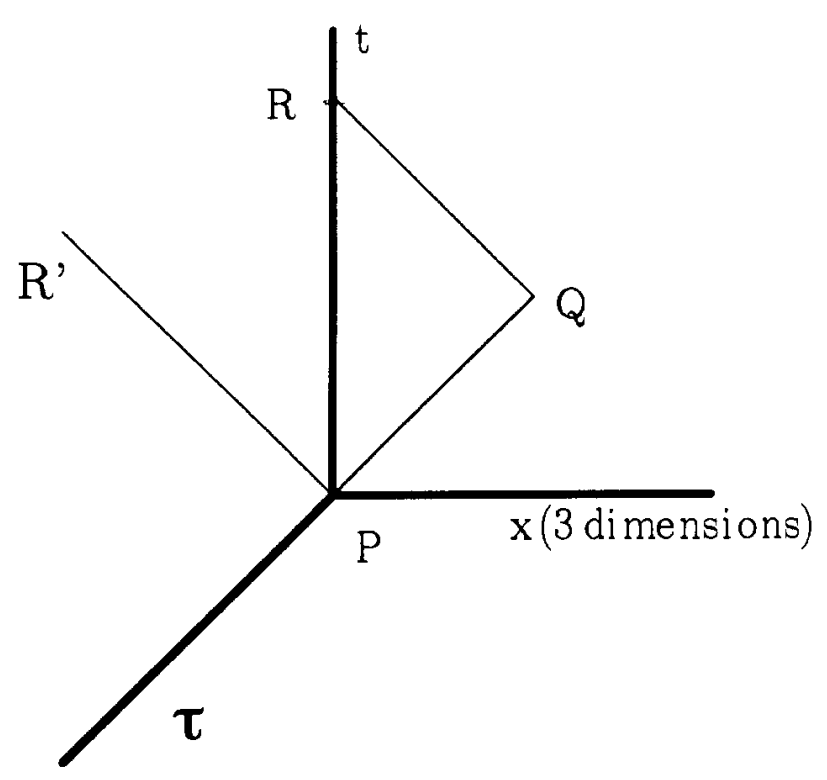

Figure 2. The twin paradox.

Let us consider the Figure 2 in order to have a clear understanding of the meaning of $\Delta x^{5}$ of a physical object as its aging. This figure may represent a vain physicist looking himself at a mirror, or the limiting case $(v \approx c)$ of the twin paradox in Special Relativity. $P R^{\prime}$ and $\mathrm{PQ}$ are two generators of a same causality-cone. PQ belongs to the light-cone (taking $v \approx c) . P R^{\prime}=\left(0,0,0, \Delta \tau, \Delta x^{5}\right)$ with $\Delta t=\Delta \tau$, while $P Q=\left(\Delta \vec{r}, \frac{\Delta t}{2}, 0\right)$ and $Q R=\left(-\Delta \vec{r}, \frac{\Delta t}{2}, 0\right) . P R^{\prime}$ is the physicist world-line on his rest frame. $R$ is the physicist image reflected (back to him) at $\mathrm{Q}$, or his twin brother returning from a trip to $\mathrm{Q}$. They meet again at the time $t=t_{R}>0$, at the same space point $(\vec{r}=0)$, from where they had departed from each other, but now with distinct fifth coordinates, $x_{R}^{5}=0$ and $x_{R^{\prime}}^{5}>0$, that represent their distinct agings.

Let us mention now a rich and interesting point of this geometry. Observe the difference between $\tau$ and $t$ in (17): they are invariant under different subgroups of isometry — $S O(3,1)$ and $O_{4}$, respectively - of the causality-cone. Both sides of

$$
(\Delta \tau)^{2}=(\Delta t)^{2}-(\Delta \vec{x})^{2}
$$

are invariant under transformation of the $S 0(1,3)$ group, that is, rotation in a Minkowski spacetime $(\vec{x}, t)$; but in

$$
(\Delta t)^{2}=(\Delta \tau)^{2}+(\Delta \vec{x})^{2}
$$


both sides are invariant under $0_{4}$, the rotation group in Euclidean 4-dimensional spacetime $(\vec{x}, \tau)$.

The use of $t$ in the place of $\tau$ as the invariant corresponds to a Wick rotation without the need of analytic continuation $[10,11], t \rightarrow i t$, and lends to it a clear physical and geometrical interpretation. Physically it means that, for an Euclidean four-dimensional spacetime, events should be labelled not by the time measured in the observer's clock, but with their local proper time, read on their local clocks. $0_{4}$ is the invariance group of the causality-cone for rotations around its t-axis. Care must be taken with the interpretation of the $0_{4}$ sub-groups involving $\tau$, as they correspond to Lorentz and conformal transformations.

For those unaccustomed to the idea of extradimensions, we remind again that the Minkowski spacetime represents the geometrization of an experimentally founded physical principle: the constancy of the speed of light. It requires that the time (up to then, just a parameter) be treated as the fourth coordinate of a four-dimensional manifold, the spacetime. We are doing here something very similar: the geometrization of causality, embodied in the relations (16) or (17). It requires a fifth coordinate with the role of a propertime.

We can now return to our initial problem, which in this geometry is pictured by an electron and its electromagnetic field (photon) in a same causality-cone, each along its own cone-generator. This picture, although simple, discards some old problems like self-interactions and their associated infinities, but this will be discussed elsewhere (see the reference [13]). Let us consider instead the metric structure of the spacetime associated to each physical object. The metric induced by (18) on (15) of a physical object, $\left(d S_{5}\right)^{2}=d x . \eta . d x-(f . d x)^{2}=$ $d x .(\eta-f f) . d x$, is given by $g_{\mu \nu}=\eta_{\mu \nu}$ for a massless field (since then $d \tau=-f . d x=0$ ), and by $g_{\mu \nu}=\eta_{\mu \nu}-f_{\mu} f_{\nu}$, with $f_{\mu}=\eta_{\mu \nu} f^{\nu}$ and $g^{\mu \nu}=\eta^{\mu \nu}+\frac{f^{\mu} f^{\nu}}{2}$ for massive physical objects. The distinct causality requirements of massive and of massless fields and particles are, therefore, represented by immersions with distinct metric structures. They can both be written in a single expression (using either $f^{2}=0$ or $f^{2}=-1$ for, respectively massless and massive objects):

$$
g_{\mu \nu}=\eta_{\mu \nu}+f^{2} f_{\mu} f_{\nu}
$$

$$
g^{\mu \nu}=\eta^{\mu \nu}-f^{2} \frac{f^{\mu} f^{\nu}}{1+\left(f^{2}\right)^{2}} .
$$

At this point we can understand that a plus sign in front of $(\Delta \tau)^{2}$ of the line element (15) would imply on $\Delta \mathcal{S}_{5} \equiv 0$ for all physical object and would induce $g_{\mu \nu}=\eta_{\mu \nu}+f_{\mu} f_{\nu}$, as a metric on a causality-cone of a massive object, which would not be consistent because then, $g_{\mu \nu} f^{\mu} \equiv 0$.

The existence of two distinct metric structures for a massive and a massless field invalidates (1) as the result of (6). While $T^{\mu \nu}{ }_{\nu}=0$ for $\rho>0$ remains valid in this new picture, its limit when $\rho \rightarrow 0$ is not as simple as described before because it involves now a local change of manifolds with different metric structure $(\eta \rightarrow \eta-f f)$. Physically it only makes sense! For $\rho>0$ one is dealing with electromagnetic fields (photons) for which $(8,11)$ represent the causality requirement that $\mathrm{A}(\mathrm{x}, \mathrm{t})$ and $z\left(\tau_{\text {ret }}\right)$ remain on a same light-cone, but for $\rho=0$ one has an electron, a massive particle, which must attend a completely different causality relation $(12,13)$. As a matter of fact, the limit of $\mathrm{K}$ when $\rho$ tends to zero is an indeterminacy that can be resolved with a derivative $d / d \tau$ and the L'Hospital rule:

$$
\lim _{\rho \rightarrow 0} K_{\mu}=\lim _{\rho \rightarrow 0} f_{\mu}=V^{\nu} \eta_{\nu \mu} \equiv V_{\mu}
$$

This is coherent with (9) and (14). This change of $\mathrm{K}$ to $\mathrm{V}$ was not considered in the limit passage of (7), as also, of course, the change of metric required by (19). This procedure extends, in fact, the photon causality constraint (11) to the electron; it corresponds to treating the electron as if it were a massless object. This new vision of spacetime requires a revision not only of the Lorentz-Dirac equation but of any theory of interacting fields. This will be discussed in subsequent papers. Our immediate goal has been attained with the stressing of the connections among causality violation in the Lorentz-Dirac equation and the spacetime structure.

\section{Summary and Conclusions}

The Lienard-Wiechert solutions are closely related to the Lorentz-Dirac equation, but while the first one has a well drawn picture of causality preservation, based on the light-cone structure of the Minkowski spacetime, the second one is, nonetheless, well known for its problematic causality violating solutions. For this reason, 
this equation has always been accompanied by many doubts about its validity. It has been obtained from the most diverse approaches and its uniqueness has been scrutinized and proved under very generic and acceptable conditions $[1,2]$. However, we do not endorse the apparently most accepted view that this is, after all, the correct equation and that its problems appear only when we stretch its application to situations when a quantum theory should be used instead.

With the strategy of geometrizing the Principle of Causality, that is, of transferring its implementation to the background spacetime structure, we find that a model of spacetime, more complex than the model of Minkowski, is required. It makes clear that the weak point common to all demonstrations of the LorentzDirac equation is the extrapolation for the electron of restrictions that are valid only for its electromagnetic field. The Minkowski spacetime represents just a geometrization of the Einstein postulates of Special Relativity, and so it does not contemplate the difference in the metric structure required for a more strict geometric implementation of causality. Therefore, the LorentzDirac equation is the result of imposing to the electron a causality behavior that is valid only for its electro- magnetic field. [13].

\section{References}

[1] E.G.P. Rowe, Phys. Rev. D, 12, 1576 (1975) and 18, 3639 (1978); Nuovo Cim. B73, 226 (1983).

[2] A. Lozada, J. Math. Phys. 30, 1713 (1989).

[3] S. Parrot, Relativistic Electrodynamics and Differential Geometry, Springer-Verlag, New York, (1987).

[4] J. C. Eliezer, Rev. Mod. Phys. 19, 148 (1947).

[5] J. D. Jackson Classical Electrodynamics, 2nd ed., chap. 14, John Wiley \& Sons, New York, NY (1975).

[6] C. Itzkyson, J. Zuber Quantum Field Theory, p. 43-44 McGraw-Hill, Singapore (1983).

[7] F. Rohrlich, Classical Charged Particles. Reading, Mass. (1965).

[8] C. Teitelboim, D. Villaroel, Ch.G. Van Weert, Rev. Nuovo Cim. 3, 1 (1980).

[9] J. R. Fanchi, Phys. Rev D20, 3108 (1979).

[10] R. L. Ingraham, Lettere al Nuovo Cimento 7, 846 (1973a); Il Nuovo Cimento 27B, 293 (1975).

[11] C. Itzkyson, J. Zuber Quantum Field Theory, p.300, 485, McGraw-Hill, Singapore (1983).

[12] Ta-Pei Cheng, Ling-Fong Li, Gauge Theory of Elementary Particle Physics. Clarendon Press-Oxford (1984).

[13] M. M. de Souza, J. of Phys A30, 6565 (1997). 